\title{
Remote Laboratories: Bridging University to Secondary Schools
}

\author{
A live demonstration at experiment@portugal
}

\author{
http://dx.doi.org/10.3991/ijoe.v8iS1.1902 \\ H. Fernandes, R.B. Henriques, J. Fortunato \\ Universidade Técnica de Lisboa, Lisbon, Portugal
}

\begin{abstract}
-e-lab is a remote laboratory infrastructure powered by a software framework that allows the operation and data retrieve from a remote apparatus. In this demonstration we will present the e-lab interface properties and its modus operandi, giving as well some topics of complimentary software use for data interpretation and analysis.
\end{abstract}

Index Terms-remote laboratories, secondary education, educations in physics, web 2.0, cloud computing

\section{INTRODUCTION}

The best education systems understand that teacher's qualification is the most influential factor in improving students' outcomes. However, their performance must be supported continuously at the level of training and motivation. Much of the motivation for teaching comes from the stimulus generated by their students. But in today's society, the student's motivation involves the use of emerging technology, as they early in their lives became acquaint and dependent on it. The use of online resources have a predominant impact on that motivation and most students, when they have access to the Internet, choose to study using such contents to the detriment of the classic book. The paradigm of the mentor/book is being changed by the use of online classes and/or movies where the information is concise and concentrated, giving the student a greater degree of freedom in every topic he needs.

However, on teaching physics or any other science, it is essential to create a bond of trust in what is learned through online resources. The use of "seals" settled over the sources of information improves contents credibility meant for public perception and should be an important factor in the decision to select it. On the other hand, the ordinary simulations (applets) often used to interpret contents more easily, belong to the "theory" class because they leverage on mathematical models. Whereas the practical activity (the "observation") is the main source of credit for science, the teaching of science in particular always need the comparison between this "observation" and theory. That's where confidence comes from.

e-lab [1] is the bridge between these two concepts: (i) incorporates the latest technology for distance learning, allowing to perform real experiments in a fully robotic remote environment and moreover (ii) allows to watch them via video as well as to collect the experiment data set. These two facts create the correct confidence on the users of its real existence and after fitting the theoretical model allow the full trust in the theory in support of the experimental evidence.

\section{THE RENEWED E-LAB}

In operation at IST since 2001, e-lab recently underwent a usability study, currently offering a simpler and more concise to use graphic user interface, allowing an immediate and easy access to the required laboratory. The contents are supported by the portal e-escola [2]. Having been used in the basic university physics disciplines of Bolonha's first cycle of studies, it was established at the present time an extension of content to cover also the secondary education level, with some experiences being specially adapted for this purpose as well as their online content.

In the other end a new recent evolution is the adaptation of e-lab to post-graduated studies. Effectively Europe is facing a new age where universities are learning the importance of cooperating among themselves to achieve the necessary "critical mass" to be able to teach very specialized courses. As an example we can cite the first European course in nuclear "fusion science and engineering": as many students are apart and they need to accomplish some credits in laboratories, Fusenet will support a few remote laboratories where advanced experiments on plasma physics will be available.

\section{DEMONSTRATION PROPOSAL}

The proposed demonstration to be arranged at experiment@portugal should cover some examples of remote access to some e-lab laboratories presently available, giving to the users the experience and control of them. In parallel, some data analysis based on MSExcel and Origin will take place to demonstrate how this complementary computer aided tools can help on the underlying physics interpretation. This presentation will be done with 2 wide screens connected to a dual VGA to demonstrate a live classroom illustration. 


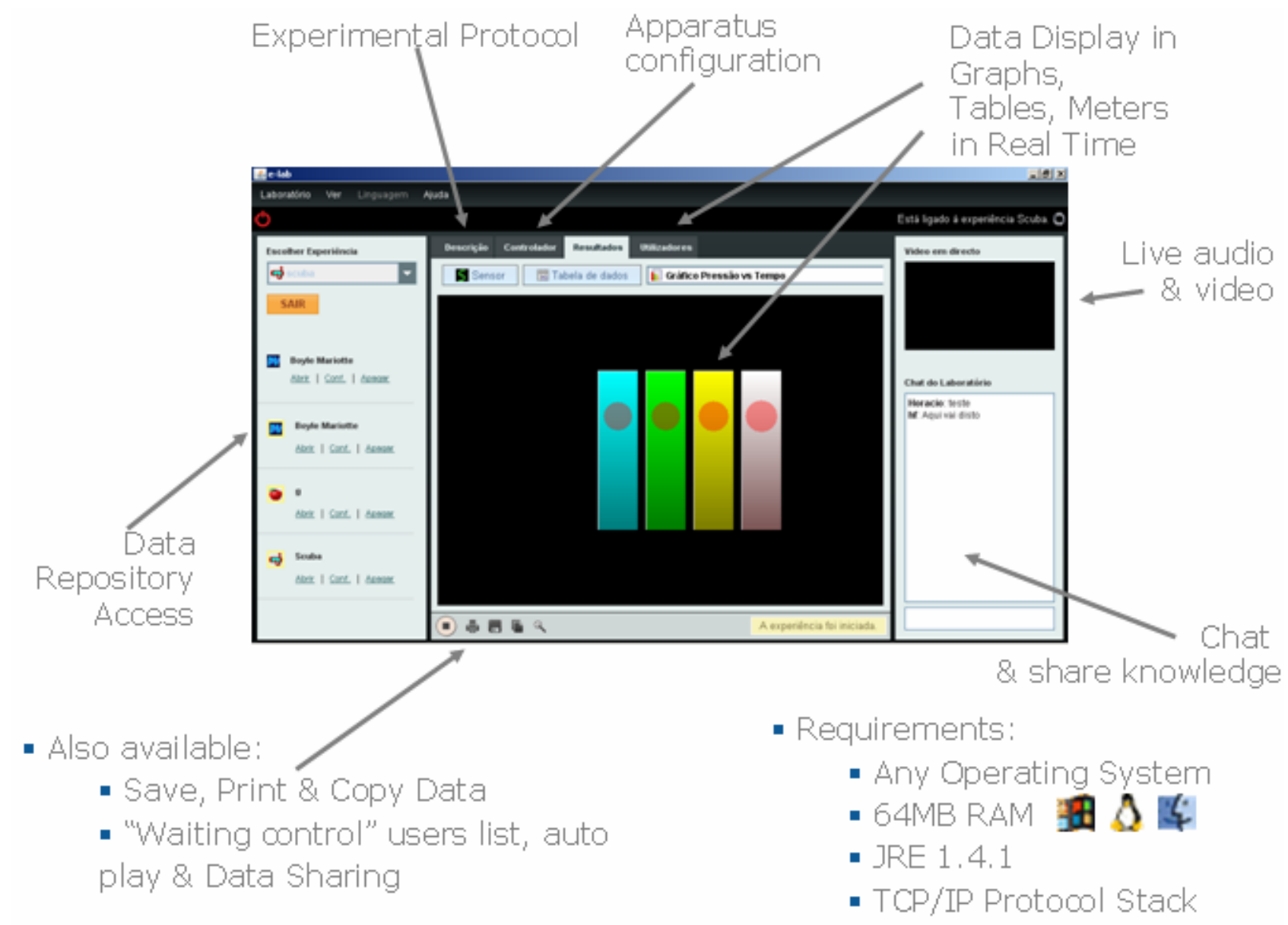

Figure 1. An example of the e-lab client GUI showing the diferent activity zones: Chat, experiment controler, video, repository, experiment selector directory, fast buttons and tab navigation panel.

The dual display will allow to (i) show the live control of an experiment with (ii) the current video being displayed in the second monitor using QuickTime or VLC (iii) and running in parallel the data analysis software tools (iv) were passing some graphical data into text documents.

E-lab have proven to be a platform capable to cover experiments from basic school up to pos-graduated education and can manage a great variety of interfaces to accommodate almost any class of laboratories. We hope to offer at experiment@portugal a good view of its potentialities and practice.

\section{ACKNOWLEDGMENT}

e-lab is a joint undertake of many people most of them regular students at IST Physics Engineering MsC which voluntary contribute to the e-lab development. For them the author leaves here a particular recognition and gratitude.

\section{REFERENCES}

[1] http://e-lab.ist.eu

[2] http://www.e-escola.pt/elab_exp.asp

\section{AUTHORS}

H. Fernandes, R.B. Henriques, and J. Fortunato are with the Instituto de Plasmas e Fusão Nuclear - Instituto Superior Técnico - Universidade Técnica de Lisboa, P1049-001 Lisboa, Portugal, (e-mail: hf@ipfn.ist.utl.pt)

This work was supported by Calouste Gulbenkian Foundation, Lisbon, Portugal. And by the European Union and Instituto Superior Técnico. It was also received partial funding from PosConhecimento and from DGIDC (General Department of Innovation and Curriculum Development), Ministry of Education. Received 1 December 2011. Published as resubmitted by the authors 20 January 2012. 